\title{
Techniques to perform robotic left adrenalectomy in the obese patient
}

\author{
Ajay V. Maker ${ }^{1,2} \cdot$ Vijay K. Maker ${ }^{2,3}$
}

Received: 5 January 2016/Accepted: 11 June 2016/Published online: 7 July 2016

(C) Springer Science+Business Media New York 2016

\begin{abstract}
Introduction Minimally invasive adrenalectomy may be associated with reduction in postoperative pain, morbidity, and length of stay and, as a result, has become a preferred approach for many adrenal tumors. Left-sided adrenal tumors, however, are particularly challenging to address in the morbidly obese patient due to difficulties in maintaining exposure and dissection. The robotic platform offers instruments with greater degrees of freedom that aid in retraction and dissection, especially of the adrenal vein, but fixed patient positioning and the large distance needed between patient ports to avoid arm collisions can be restrictive in patients with a large amount of retroperitoneal fat and small working space.

Methods/Results We demonstrate robotic left adrenalectomy (RLA) in a consecutive series of patients with a
\end{abstract}

This video was presented, in part, as an oral presentation at the American College of Surgeons Clinical Congress 2015, Chicago, IL.

Electronic supplementary material The online version of this article (doi:10.1007/s00464-016-5049-9) contains supplementary material, which is available to authorized users.

Ajay V. Maker

amaker@uic.edu

1 Division of Surgical Oncology, Department of Surgery, College of Medicine, University of Illinois at Chicago, $835 \mathrm{~S}$. Wolcott Ave, MC790, Chicago, IL 60612, USA

2 Creticos Cancer Center, Advocate Illinois Masonic Medical Center, Chicago, IL 60657, USA

3 Department of Surgery, College of Medicine, University of Illinois at Chicago, Chicago, IL 60612, USA mean weight of $99 \mathrm{~kg}$ and mean BMI of 36 . Techniques to safely and efficiently perform RLA in obese patients are stepwise demonstrated, including (1) Patient positioning, (2) Management of the pannus, (3) Customized port placement, (4) Medial retraction of the pancreas, (5) Finding the left adrenal vein, and (6) Management of bleeding. Intraoperative videos from multiple patients also show surgical pitfalls, examples of poor port placement, arm collisions, alternative approaches to the vein, and techniques to control unexpected bleeding. All patients in the series underwent successful RLA with negative margins, no major intra- or postoperative complications, and discharge on POD 1-2.

Conclusion Though poor exposure due to patient body habitus is a relative contraindication, even large left-sided adrenal tumors can be safely approached robotically while adhering to oncologic principles, as is demonstrated in this video.

Acknowledgments Dr. Maker is supported by the NIH/NCI K08 CA190855.

\section{Compliance with ethical standards}

Disclosure Ajay V. Maker and Vijay K. Maker have no conflicts of interest or financial ties to disclose. 